\title{
The study of sperm head vacuoles using deep learning algorithm and its correlation with protamine mRNA ratio
}

Fatemeh Ghasemian ( $\square$ ghasemian.21@gmail.com )

University of Guilan Faculty of Basic Sciences https://orcid.org/0000-0001-9284-6545

Mohammad Hadi Bahadori

Guilan University of Medical Sciences

Seyedeh Zahra Hosseini Kolkooh

Guilan University of Medical Sciences

Maryam Esmaeili

Guilan University of Medical Sciences

\section{Research}

Keywords: Human sperm, Vacuole, Protamination, Pregnancy, Deep learning algorithm

Posted Date: January 20th, 2020

DOI: https://doi.org/10.21203/rs.2.21320/v1

License: (c) (i) This work is licensed under a Creative Commons Attribution 4.0 International License.

Read Full License 


\section{Abstract}

\section{Background}

As regards the routine semen analysis is not sufficient to assess male fertility status, is it necessary to use other morphological sperm examination that may be more relevant in regard to the promotion of assisted reproduction outcomes? This study was designed for examination of sperm vacuole characteristics, its association with other sperm parameters and protamine 1 to protamine 2 ratio, and predict assisted pregnancy outcomes.

Methods

98 Semen samples from subfertile men were classified based on Vanderzwalmen's criteria as follows: grade I, no vacuoles; grade II, $\leq 2$ small vacuoles; grade III, $\geq 1$ large vacuole; grade IV, large vacuole with other abnormalities. The location, frequency and size of vacuoles were assessed using high magnification, a deep learning algorithm, and scanning electron microscope methods. The chromatin integrity (toluidine blue staining), condensation status (aniline blue), viability and acrosome integrity (triple staining), and protamination status (CMA3 staining) was evaluated for vacuolated samples. Protamine 1 and protamine 2 gene expression was analyzed by quantitative real-time PCR. The assisted reproduction outcomes were also followed for each cycle.

Results

The results show a significant correlation between the vacuole size (III and IV) and abnormal sperm chromatin condensation $(p<0.05)$, and protamine-deficient $(p<0.05)$. The percentage of reacted acrosomes was significantly higher in spermatozoa with grades III and IV compared with normal group $(p<0.05)$. A high protamine mRNA ratio ( $p r m-2$ was underexpressed) was observed in the vacuolated spermatozoa with grade IV $(p<0.01)$. The sperm head vacuole was negatively associated with the fertilization rate $(p<0.01)$ under IVF cycles. This association was also significantly observed in pregnancy and live birth rate in the groups with grade III and IV $(P<0.05)$.

Conclusions

The results of our study highlight the importance of follow up of more sperm parameters such as sperm head vacuole characteristics, because may reflect protamine-deficient and poor IVF/ICSI outcomes.

\section{Introduction}

Forasmuch as normal sperm parameters were found in almost $15 \%$ of infertile males, the routine semen analysis is not sufficient to assess male fertility status (Perdrix et al, 2011). Nowadays, several assays have been suggested to progress the male infertility diagnosis with more details (lewis, 2007), which can be included sperm morphology (using light and electron microscopy), nucleus assessment (chromatin integrity and condensation, protamination status, aneuploidy), and the function of sperm (e.g., motility, 
viability, oxidative stress, acrosome reaction, sperm-zona pellucida interaction) (Perdrix et al, 2011). In addition, the evaluation of the detailed morphology of motile sperm was performed in real-time at a high magnification (up to $\times 6600$ ) which is called the motile sperm organelle morphology examination (MSOME). In fact, MSOME is seen sperm morphology with more details, which ones are not appeared when viewed at $\times 400$ or $\times 200$ magnifications (Bartoov et al., 2002).

Sperm head vacuole has been defined as one of the most important of sperm abnormalities (Bartoov et al., 2002). But the origin and nature of vacuole remains unknown. Sperm morphology, especially head vacuoles, proved to have a major effect on assisted reproductive techniques (ARTs) outcomes (Bartoov et al., 2002; Vanderzwalmen et al., 2008; Cassuto et al., 2009; Knez et al., 2011; Knez et al., 2013). Therefore, the selection of 'good' spermatozoa prior to intracytoplasmic sperm injection (ICSI) is correlated to the best assisted reproductive outcomes. The presence of morphologically and morphometrically normal head (Bartoov et al., 2002; Berkovitz et al., 2005; Hazout et al., 2006; Antinori et al., 2008) lack of vacuoles (Berkovitz et al., 2006) or fewer than two small vacuoles (Cassuto et al., 2009) is determined a 'good' spermatozoa (Boitrelle et al., 2011).

In addition, the successful fertilization is affected by competent sperm, the most important of which is replacement of DNA-binding histones by protamines (Steger et al., 2011; Rogenhofer et al., 2013). Recently, an association between improper protamine mRNA/protein ratio and male infertility has been found (de Mateo et al., 2009; Hammoud et al., 2009; Depa-Martynow et al., 2012; Rogenhofer et al., 2013). This ratio is known as a suitable biomarker for successful fertilization (Carrell and Liu, 2001; Aoki et al., 2006; DepaMartynow et al., 2012). The relative ratio of protamine-1 to protamine-2 is almost at a 1:1 ratio (Balhorn et al., 1988). A range from 0.5 to 1.4 has been determined in normozoospermia samples (Nanassy et al., 2011). The correlation between male infertility and abnormal protamine mRNA ratio has been found (Rogenhofer et al., 2013). However, it should be further investigated whether the protamine mRNA ratio is impacted by vacuolated spermatozoa percentage, and correlated with fertilization, embryo development and pregnancy rates during in-vitro fertilization (IVF) or ICSI cycles. Therefore, the focus on sperm genomic quality and its association with improvement in assisted reproductive outcomes has been one of the most important goals in recent years.

However, the significant of vacuoles, as well as their effects on fertility potential of sperm, protamine mRNA ratio, and assisted reproductive outcomes (e.g., fertilization rate, embryo development, and pregnancy rate) are not clear. Therefore, to establish a correlation between vacuole(s) and the protamine1 to protamine-2 mRNA ratio and assisted reproductive outcomes, we used deep learning algorithm to select the 'good' spermatozoa. We then compared the protamine-1 to protamine-2 mRNA ratio among spermatozoa with different vacuole grades. In addition, the acrosome reaction, chromatin condensation and integrity, protamination status, and fertilizing capacity of semen samples with different degrees of vacuolated spermatozoa were studied during ICSI/IVF cycles.

\section{Materials And Methods}




\section{Participants}

A total of 309 specimens was collected from subfertile men (age 22-38 yrs) who visited in Alzahra hospital (IVF center), Rasht, between May 2018 to September 2019. This study was approved by the Guilan University of Medical Sciences committee. In addition, the informed consent was obtained from all the selected participants in the present study. The couples were treated under ICSI or IVF cycles according to spermocytogram, previous failed cycles, and woman age. The semen samples were analyzed and the semen samples with vacuolated sperms were included in this study ( $n=98$ couples). The exclusion criteria were the female factor infertility, maternal age higher than 40 years, and patients with less than three oocytes. The couples with severe male factor infertility were also excluded from this study to remove their effects on sperm quality.

The semen samples were collected via masturbation after three to four days of sexual abstinence. The semen samples were analyzed according to the World Health Organization (WHO) criteria (WHO, 2010). The semen parameters such as $\mathrm{pH}$, volume, motility, morphology, concentration, viability were assessed. Using high magnification ( $\times 1000$ ), a novel deep learning algorithm (Javadi and Mirroshandel, 2019) and scanning electron microscopy (SEM), the semen samples were categorized into four groups according to Vanderzwalmen's criteria as follows: grade I) no vacuoles; grade II) $\leq 2$ small vacuoles (which occupy < $4 \%$ of the head's area); grade III) more than two small vacuoles or $\geq 1$ large vacuole (which occupy between $13 \%$ to $50 \%$ of the head's surface area); and grade IV) large vacuole with other abnormalities (Figure 1) (Vanderzwalmen et al, 2008).

\section{Assay using a novel deep learning algorithm}

Using deep learning algorithm, sperm morphology, especially vacuole was analyzed. This algorithm was performed with a high accuracy $(94.65 \%)$ to detect sperm's vacuoles. In addition, this method worked very fast and categorized sperm images in real-time. Therefore, the classification of spermatozoa was done using this algorithm in line with the results of high magnification $(\times 1000)$ and SEM images (Javadi and Mirroshandel, 2019).

\section{Scanning electron microscopy (SEM)}

For correct measurement of sperm vacuole, each semen samples were evaluated by scanning electron microscopy (SEM) to observe the smallest details. The fixation of semen samples washed via density gradient centrifugation method was performed using Karnovsky solution for $30 \mathrm{~min}$ at $4{ }^{\circ} \mathrm{C}$. After centrifugation at $4,000 \times \mathrm{g}$ for $30 \mathrm{~min}$, the samples were washed and the postfixation was done with $1 \%$ osmium tetroxide for 30 min. Afterwards, the ascending degrees of ethanol $(50 \%, 70 \%, 80 \%, 90 \%, 96 \%$, and absolute alcohol) were used to dehydrate. The drying was performed at a critical point (Balzers CPD010). The specimens coated with gold (Balzers MED-010) were examined in a Philips FEM 515 scanning electron microscope.

\section{Sperm chromatin assays}




\section{Toluidine blue stain}

The abnormality in the sperm chromatin structure was distinguished using toluidine blue (TB) staining. In this way, the air-dried smears on silane-coated slides were fixed in $96 \%$ ethanol-acetone medium (1:1) at $4^{\circ} \mathrm{C}$ for 1 hour. To hydrolysis, slides were put in $0.1 \mathrm{~N} \mathrm{HCl}$ at $4^{\circ} \mathrm{C}$ for $5 \mathrm{~min}$, then were washed. The staining was done with $0.05 \%$ toluidine blue (TB, in $50 \%$ Mcllvaine's citrate phosphate buffer, $\mathrm{pH} 3.5$, Merck) for $5 \mathrm{~min}$ at room temperature (RT). Approximately 200 sperms were evaluated in each slide using a light microscope. The observation of light blue or deep violet/purple heads is the sign of existence normal or abnormal chromatin structure, respectively.

\section{Aniline blue stain}

The adhesion between lysine residues of histones and aniline blue (AB) stain were detected the abnormal condensation of sperm chromatin. Briefly, the smears were fixed in $4 \%$ formalin (Junsei Chemical, Tokyo, Japan). After washing, the slides were stained in 5\% AB (Sigma-Aldrich Co., St. Louis, MO, USA) in a solution of $4 \%$ acetic acid ( $\mathrm{pH} 3.5$ ) for $5 \mathrm{~min}$ at RT. Almost 200 spermatozoa in each slide were observed under a light microscope. The sperms with dark-blue or colorless head were considered as abnormal or normal chromatin condensation, respectively.

\section{Acrosome reaction assessment}

The acrosome status (reacted acrosome or intact acrosome) was evaluated using triple staining and according to Talbot and Chacon method (Talbot \& Chacon, 1981). In brief, sperms were put in $2 \%$ trypan blue (1:1), incubated at $37^{\circ} \mathrm{C}$ for $15 \mathrm{~min}$, and centrifuged at $600 \times \mathrm{g}$ for $5-10 \mathrm{~min}$ ). Then, the sperm pellet was washed to obtain a clear/ pale blue supernatant. The sperms were fixed using glutaraldehyde ( $3 \%$ glutaraldehyde in $0.1 \mathrm{M}$ cacodylate buffer at $\mathrm{pH} 7.4$ ) for 30 to $60 \mathrm{~min}$, centrifuged at $6000 \times \mathrm{g}$ for $5 \mathrm{~min}$, and stained with Bismark brown $\mathrm{Y}$ at $40{ }^{\circ} \mathrm{C}$ for $5 \mathrm{~min}$. The staining with Rose Bengal was done at $24^{\circ} \mathrm{C}$ for 20-45 min. Then, the smears were washed (in water), dehydrated (in an ascending degree of alcohol), and cleared (in xylene). At the end, almost 300 sperms in each slide were examined under a light microscope. Four staining templates were seen as follows:

i) Dead sperm and intact acrosome as dark-blue post-acrosomal regions and pink acrosomes, respectively, ii) Dead sperm and degenerated acrosome as dark-blue post-acrosomal regions and blue/white acrosomes, respectively, iii) Alive sperm and intact acrosome as light brown postacrosomal regions and pink acrosomes, respectively, and iv) Alive sperm and degenerated acrosome as light brown post-acrosomal regions and blue/white acrosomes, respectively.

\section{Determination of sperm deprotamination by chromomycin A3}

The sperm protamination was distinguished by chromomycin A3 (CMA3) stain (Figure 2) as a detector of guanosine-cytosine-rich sequence. The fixation of air-dried smears was done in methanol/glacial acetic acid (3:1) for $20 \mathrm{~min}$ at $4{ }^{\circ} \mathrm{C}$. Then, the slides were put in $100 \mu \mathrm{l} \mathrm{CMA3} \mathrm{solution}(0.25 \mathrm{mg} / \mathrm{ml}$ CMA3 in 
Mcllvaine's buffer, containing $10 \mu \mathrm{m} \mathrm{MgCl}_{2}$ ) for $20 \mathrm{~min}$. The sperms with dull yellow staining (CMA3 negative) and bright yellow fluorescence (CMA3 positive) were considered as normal and abnormal chromatin protamination, respectively.

\section{RNA extraction and first strand cDNA synthesis}

RNA extraction was performed using the RNeasy Mini kit (Roche Molecular Biochemicals, Mannheim, Germany) and stored at $-80^{\circ} \mathrm{C}$. The first strand CDNA synthesis was done the CDNA kit (Thermo Scientific, EU), according to the manufacturer's protocol at $42{ }^{\circ} \mathrm{C}$ for $60 \mathrm{~min}$, and stored at $-20^{\circ} \mathrm{C}$.

\section{Real-time quantitative reverse transcription polymerase chain reaction}

Real-time qRT-PCR was done to quantify mRNA transcript levels of protamine-1 (prm1) and protamine-2 (prm2) genes. The primer pairs of prm1, prm2, and GAPDH (housekeeping) genes were designed by GenBank at NCBI (Table 1). The analyzing gene expression was conducted by real time thermal cycler (Applied Biosystems, Foster City, USA) and QuantiTect SYBR Green RT-PCR kit (Applied Biosystems) was also used for amplifying the reference and the target genes ( $5 \mathrm{ml}$ cDNA per sample) in the same run. The conditions were $95^{\circ} \mathrm{C}$ for 5 min (holding step), $95^{\circ} \mathrm{C}$ for $15 \mathrm{~s}, 58^{\circ} \mathrm{C}$ for $30 \mathrm{~s}$, and $72{ }^{\circ} \mathrm{C}$ for $15 \mathrm{~s}$ (cycling stem), which was followed by a melt curve step at $95^{\circ} \mathrm{C}$ for $15 \mathrm{sec}, 60^{\circ} \mathrm{C}$ for $1 \mathrm{~min}$, and $95^{\circ} \mathrm{C}$ for $15 \mathrm{sec}$. All PCR amplifications were carried out in triple and mean values were calculated. Determining of relative quantitation for target genes was performed using $\Delta \Delta C T$ method.

Table 1

Designed primer sequences used for real-time qRT-PCR.

\begin{tabular}{|c|c|c|c|c|}
\hline Genes & Primer pair sequence $(5 \rrbracket-3 \rrbracket)$ & $\begin{array}{l}\text { Annealing } \\
\text { Temperature }\end{array}$ & $\begin{array}{l}\text { Product } \\
\text { Length (bp) }\end{array}$ & $\begin{array}{l}\text { Size } \\
\text { (bp) }\end{array}$ \\
\hline \multirow[t]{2}{*}{ Protamine-1 } & F 5区 ACTAGATGCACAGAATAGCAA 3》 & 54.0 & \multirow[t]{2}{*}{134} & 21 \\
\hline & R 5囚 GTGGCATTGTTCCTTAGCAG 3® & 54.3 & & 20 \\
\hline \multirow[t]{2}{*}{ Protamine-2 } & F 5】 CAGCCTCAATCCAGAACCTCC 3囚 & 60.5 & \multirow[t]{2}{*}{108} & 21 \\
\hline & $\begin{array}{l}\text { R 5区 CTCGCGTTCATGGTCTTGTCC } \\
3 \rrbracket\end{array}$ & 60.6 & & 21 \\
\hline \multirow[t]{2}{*}{$\begin{array}{l}\text { GAPDH } \\
\text { (endogenous) }\end{array}$} & $\begin{array}{l}\mathrm{F} \\
\text { 5囚CAAGGTCATCCATGACAACTTTG3囚 }\end{array}$ & 58.2 & \multirow[t]{2}{*}{183} & 23 \\
\hline & $\begin{array}{l}\mathrm{R} \\
5 \rrbracket G T C C A C C A C C C T G T T G C T G T A G 3 \rrbracket\end{array}$ & 63.08 & & 22 \\
\hline
\end{tabular}

\section{ICSI and IVF laboratory procedures}

Semen samples were collected via masturbation on the oocyte retrieval day. A period of three to four days of the ejaculatory abstinence was considered. After liquefaction of semen samples, the washing of 
semen was done by density gradient centrifugation method (90 and $45 \%$ SpermGrade, Vitrolife, Sweden). The evaluation of semen parameters was performed according to the World Health Organization (WHO, 2010) criteria. These parameters including volume, motility, concentration, morphology, and $\mathrm{pH}$ can be mentioned. The washed semen samples were incubated at $37^{\circ} \mathrm{C}$ in a $5 \% \mathrm{CO}_{2}$ atmosphere up to three to four hours for performance of ICSI or IVF procedures. Treatment procedure (ICSI/IVF) was selected based on the sperm concentration, morphology, motility, infertility etiology, patient's history, and female age.

A first selection of motile spermatozoa in the poly-vinyl pyrrolidone (PVP) drop was performed at $\times 400$ magnification during ICSI cycle. Photo-documented was analyzed using a novel deep learning algorithm as real-time and used for the grading. The best spermatozoa selected was immobilized and injected as described previously for conventional ICSI (Vanderzwalmen et al., 1996) at $\times 200$ and $\times 400$ magnifications. The injected oocytes were incubated. The selected spermatozoa were also assessed at ×1000 magnification (under a Hoffman modulation contrast) in line with deep learning algorithm' results.

If possible, the selection of the best spermatozoa without vacuoles and other abnormalities was considered for injection into the oocytes. Depending on the rate of sperm morphology impairment, it may take approximately 2 to $15 \mathrm{~min}$ to selection the best spermatozoa. After this time, the second-best spermatozoa with the least number of vacuoles and/or other abnormalities were selected for injection. The number of oocytes that have to be injected also affected on the research for normal spermatozoa.

Then, the analysis of fertilization was done by observing of two pronuclei in 16-18 hours after ICSI or IVF. In addition, the evaluation of embryo development rate and embryo transfer (ET) was performed at four to five days after fertilization.

\section{Statistical analysis}

Using the Chi-square test, the relationship between vacuolization grade and other semen parameters was analyzed. Fisher's exact test was used to assess the statistical correlation of vacuolization grade with clinical variables. The categorized protamine- 1 to protamine-2 ratios were analyzed using the Bonferroniadjusted Mann-Whitney U-test. Statistical analysis was done using SPSS version 20 (IBM, Armonk, NY, USA) and the values with $P<0.05$ were considered significant. To evaluate the effect of each feature/factor of vacuole (size, location and frequency) on the sperm parameters and ART outcomes, CorrelationAttributeEval and Ranker modules of WEKA software were used.

\section{Results}

\section{Grouping of vacuolated spermatozoa}

The classification of spermatozoa was done using a novel deep learning algorithm (Javadi and Mirroshandel, 2019). The results of this algorithm were similar to the results of high magnification ( $x$ 1000). In addition, scanning electron microscope (SEM) images showed more details of vacuole (size, location, and frequency) in the semen samples of each patient (Fig. 3). Therefore, grouping of samples 
was done very precisely based on the novel deep learning algorithm, high magnification $(\times 1000)$, and the SEM images.

\section{Vacuolization and sperm parameters}

The data of Table 2 show significant difference in the rate of abnormal condensation of chromatin sperm (AB staining) in the grade IV $(p<0.05)$ in comparison to control group (grade I). There was no significant difference in the viability and abnormality of the DNA structure of vacuolated spermatozoa among different grades $(p>0.05)$.

The presence of bright yellow fluorescence (CMA3-positive) was more frequently observed in spermatozoa with a large nuclear vacuole (LNV) than other groups (2336/4200; 55.6\% vs. $621 / 1500 ; 41.4 \%$, respectively), reflecting a higher percentage of sperm deprotamination in vacuolated spermatozoa with grade III $(p<0.05)$ and IV $(p<0.01)$. Also, the presence of more than one small nuclear vacuole shows more abnormal chromatin deprotamination in comparison to large non-nuclear vacuole $(p<0.05)$. The percentage of reacted acrosomes (blue/white) was significantly higher in spermatozoa with non-nuclear vacuoles (grade III and IV) in comparison to other groups $(p<0.05)$.

Table 2

The effect of vacuolization grade on different sperm parameters

\begin{tabular}{|lllllll|}
\hline Vacuole grade & $\mathbf{N}$ & $\begin{array}{l}\text { CMA3 } \\
\text { (\%) }\end{array}$ & Spont. AR (\%) & $\begin{array}{l}\text { A.B. } \\
\text { (\%) }\end{array}$ & $\begin{array}{l}\text { T.B. } \\
(\%)\end{array}$ & $\begin{array}{l}\text { Viability } \\
\text { (\%) }\end{array}$ \\
\hline I & 27 & $28.3 \pm 2.9$ & $15.1 \pm 1.9$ & $22.7 \pm 2.1$ & $23.7 \pm 2.4$ & $65.45 \pm 10.08$ \\
\hline II & 24 & $32.4 \pm 2.6$ & $19.8 \pm 2.1$ & $29.5 \pm 2.2$ & $26.8 \pm 2.5$ & $59.9 \pm 9.81$ \\
\hline III & 23 & $36.1 \pm 3.2^{*}$ & $27.16 \pm 2.4^{*}$ & $32.4 \pm 3.2$ & $28.9 \pm 3.1$ & $59.02 \pm 9.07$ \\
\hline IV & 24 & $41.3 \pm 3.4^{* *}$ & $29.9 \pm 2.7^{*}$ & $38.6 \pm 3.6^{*}$ & $29.7 \pm 3.2$ & $55.14 \pm 9.14$ \\
\hline $\begin{array}{l}\text { N: Number of patients; CMA3: Chromomycin A3; Spont. AR: Spontaneously Acrosome Reaction; AB: } \\
\text { Aniline Blue; TB: Toluidine Blue. There is significant difference between chromatin protamine-deficient } \\
\text { (CMA3), spontaneously reacted acrosomes, abnormal chromatin condensation (AB staining) in the } \\
\text { spermatozoa with grade III and IV compared to control group. P value: } *<0.05, * *<0.01 \text { and } * \star *<0.001 .\end{array}$ \\
\hline
\end{tabular}

\section{The protamine mRNA ratio in the vacuolated spermatozoa from fertile and subfertile men}

The assessment of gene expression (prm1 and prm2) shows that there is a significant difference in prm1 gene expression between III (median 0.4457; $p<0.05)$ and IV ( $p<0.0001)$ groups in comparison to control group (group I), while there is no significant difference between II group (median 0.83184) compared with the control group (median 1.0201; $p=0.09$ ) (Fig. 4). In addition, the analysis of prm2 gene expression shows significant differences between groups of II (0.6623; $p<0.01)$, III (median 0.60262; $p<0.0001$ ), and IV (median 0.2772; $\mathrm{p}<0.0001$ ) compared to the control group (median 1.001; group I).

The protamine mRNA ration was evaluated between different vacuolization grades in the fertile and subfertile men. Vacuolated spermatozoa from subfertile men with grade IV (median 3.40006 $\pm 1.81 ; p<$ 
0.01 ) displayed a significant difference in the protamine mRNA ratio in comparison to control group (median 1.02 $\pm 0.81 ; p<0.01$ ).

\section{Vacuolated spermatozoa and IVF/ICSI outcomes}

The results of the influences of different grading of sperm vacuolization and normal sperm on the clinical outcomes are shown in Table 3. The fertilization rate decreased in the couples under IVF cycles who receiving sperms with grade III (39.6\%) and IV (32.4\%) compared to control group $(70.3 \% ; p<0.01)$. However, no significant difference was seen in the fertilization rate under ICSI cycles (III; $60.52 \%$ and IV; $57.2 \%$ ) in comparison to the control group (65.1\%; $p>0.05$ ). Increased levels of sperm vacuoles (grades III; $51.33 \%$ and IV; $49.17 \%$ during ICSI cycles and grades III; $55.01 \%$ and IV; $56 \%$ during IVF cycles) were also associated with decreased rate of embryo development in comparison to the control group (57\% and $68.1 \%$ under IVF or ICSI cycles, respectively; $p<0.05)$. The increased percentage of vacuolated sperms was correlated with the decreased chance of an embryo developing to the blastocysts stage. In this way, the rate of successful pregnancy was significantly decreased in the groups with vacuolated sperms (III and IV) under IVF treatment ( $28.57 \%$ and vs 21.42 , respectively, $\mathrm{p}<0.05)$ compared to the control (Grade I) group, while this outcome was also significant in ICSI group (33.3 and 20, respectively, $p<0.01)$.

Table 3

The effect of different vacuolization grade on assisted reproductive outcomes

\begin{tabular}{|c|c|c|c|c|c|}
\hline $\begin{array}{l}\text { Vacuole grade of } \\
\text { sperm }\end{array}$ & & $\begin{array}{l}\text { Fertilization } \\
\text { rate } \\
\text { (\%) }\end{array}$ & $\begin{array}{l}\text { Cleavage } \\
\text { rate } \\
(\%)\end{array}$ & $\begin{array}{l}\text { Clinical pregnancy } \\
\text { rate } \\
\text { P/ET (\%) }\end{array}$ & $\begin{array}{l}\text { Live birth } \\
\text { LB/IE (\%) }\end{array}$ \\
\hline \multirow[t]{2}{*}{ I } & ICSI & 65.1 & 57 & $5 / 12(41.66)$ & $3 / 5(60)$ \\
\hline & IVF & 70.3 & 68.1 & $7 / 15$ (46.66) & $4 / 7(57.1)$ \\
\hline \multirow[t]{2}{*}{ II } & ICSI & 53.26 & 55.31 & 4/11 (36.36) & $2 / 4(50)$ \\
\hline & IVF & 60.03 & 62.32 & 5/13 (38.46) & $3 / 5(60)$ \\
\hline \multirow[t]{2}{*}{ III } & ICSI & 60.52 & $51.33^{*}$ & 3/9 (33.33)* & $\begin{array}{l}1 / 3 \\
(33.3)^{\star}\end{array}$ \\
\hline & IVF & 39.6 ** & $55.01 *$ & $4 / 14(28.57)^{\star}$ & $1 / 4(25)^{*}$ \\
\hline \multirow[t]{2}{*}{ IV } & ICSI & 57.2 & $49.17^{\star}$ & $3 / 10(30)^{\star \star}$ & $\begin{array}{l}1 / 3 \\
(33.3)^{*}\end{array}$ \\
\hline & IVF & $32.4^{\star \star}$ & $56^{*}$ & $3 / 14(21.42)^{\star \star}$ & $\begin{array}{l}1 / 3 \\
(33.3)^{*}\end{array}$ \\
\hline \multicolumn{6}{|c|}{$\begin{array}{l}\text { ICSI: Intracytoplasmic sperm injection; IVF: In vitro fertilization; P: Positive cycle; ET: Embryo transfer; } \\
\text { LB: Live Birth; IE: Implanted Embryo. There is significant difference between IVF/ICSI outcomes (such } \\
\text { as fertilization rate, embryo development rate, clinical pregnancy rate, and live birth rate) and cycle } \\
\text { treated with vacuolated spermatozoa with grades of III and IV. P value: }{ }^{*}<0.05, * *<0.01 \text { and } * * *<0.001 \text {. }\end{array}$} \\
\hline
\end{tabular}




\section{Factors Ranking}

Another important experiment is to measure the effect of each feature/factor of vacuole, including size, location and frequency on male fertility potential. In this way, CorrelationAttributeEval and Ranker modules of WEKA software were used. Table 4 shows the effects of different features/factors. The effect of vacuole location (nuclear) weighed more than the effect of other parameters on pregnancy.

Table 4

The effect of different features of vacuoles on male fertility potential

\begin{tabular}{|lllllll|}
\hline Feature & $\begin{array}{l}\text { Protamination } \\
\text { status }\end{array}$ & $\begin{array}{l}\text { Protamine } \\
\text { ratio }\end{array}$ & $\begin{array}{l}\text { Chromatin } \\
\text { condensation }\end{array}$ & $\begin{array}{l}\text { Acrosome } \\
\text { reaction }\end{array}$ & Fertilization & Pregnancy \\
\hline $\begin{array}{l}\text { Nuclear } \\
\text { location }\end{array}$ & 0.0812 & 0.0752 & 0.0537 & 0.0529 & 0.0832 & $0.0875^{*}$ \\
\hline Number & 0.0689 & 0.0543 & 0.0312 & 0.0241 & 0.0776 & 0.07601 \\
\hline Size & 0.0567 & 0.0487 & 0.0192 & 0.0138 & 0.0617 & 0.0651 \\
\hline
\end{tabular}

\section{Discussion}

A novel insight was provided in this study that how vacuolization affects sperm fertility potential and is a better predictor of IVF/ICSI outcomes following evaluation of sperm using high magnification, deep learning algorithm, and SEM images. The results of this study show that variations in vacuole with higher size, greater frequency, and nuclear location were seen in protamine-deficient sperms (CMA3 positive and aberrant prm1 and prm2 gene expression) than in non-deficient ones. In addition, the presence of nonnuclear vacuole leads to increased immature acrosome reaction and decreased fertilization rate under IVF cycles.

Although many studies have indicated diagnostic limitations during the routine semen analysis for the infertile couples, this analysis is still performed in many clinical practices (López et al., 2013). However, this conventional semen analysis does not recognize the subtle abnormalities in the male genome, DNA structure and condensation (López et al., 2013). The abnormalities in chromatin structure and condensation is known to be correlated with numerous indicators of assisted reproductive outcomes, including fertilization, embryo development rate and quality, pregnancy and spontaneous miscarriage (Larson-Cook et al., 2003; Seli et al., 2004; López et al., 2013). Although it is determined that human sperms have a highly dynamic and key roles in embryo development, the utility of more detail analysis of sperm is still a matter of debate (Sergerie et al., 2005; Cassuto et al., 2012; López et al., 2013). In the present study, the predictive value of vacuolated sperm testing was distinguished between potentially pregnant and not potentially pregnant couples under IVF or ICSI cycles. As mentioned above, these poor outcomes may have related to abnormalities of chromatin condensation and sperm deprotamination (CMA3 positive with aberrant prm1 and prm2 gene expression). While, the cause of abnormal sperm 
chromatin condensation is still unclear. The results of this study suggest a direct correlation between sperm nuclear vacuolization and abnormalities in sperm chromatin packaging. It seems that the contribution of the immune seminal cells, mature sperms and immature germ cells lead to the production of reactive oxygen species that can cause vacuolated head in sperms (López et al., 2013). It has been also reported that poor chromatin condensation and aneuploidy could be observed in spermatozoa with large vacuoles (Perdrix et al., 2011).

In this study, the protamine mRNA ratio in the fertile men was obtained median $0.793 \pm 0.221$ from vacuolated spermatozoa with different grade. In addition, this ratio from subfertile men was $0.739 \pm$ 0.212 and $3.400 \pm 1.281$ in the vacuolated spermatozoa with grades III and IV, respectively. While, the protamine $m R N A$ ratio has been reported in the previous studies as follows: $0.83 \pm 0.05$ (Carrell and Liu, 2001; $n=50$ ), $1.3 \pm 0.1$ (de Mateo et al., 2009; $n=12$ ) and $0.98 \pm 0.02$ (Nanassy et al., 2011; $n=77$ ), a range of 0.54 to 1.43 of the protamine ratio has been reported in normozoospermic men (Nanassy et al., 2011). Therefore, this study's outcomes indicate that vacuolization affects negatively the protamine ratio in the subfertile men. So that, a low protamine ratio was seen in the vacuolated spermatozoa with grade III (protamine-1 was underexpressed). Also, a high protamine ratio was observed in the vacuolated spermatozoa with grade IV (normal expression of protamine-1 and underexpression of protamine-2). Aoki et al. (2006) reported prm-1 under-expression and prm-2 over-expression in infertile patients with a low protamine ratio. On the other hand, in patients with a high protamine ratio, prm-2 was underexpressed and prm-1 has normal expression. Numerous studies also indicated a significantly aberrant protamine ratio in infertile men (Carrell and Liu, 2001; Mengual et al., 2003; Nasr-Esfahani et al., 2004; Aoki et al., 2005; De Mateo et al., 2009) and our result is in line with the reported ratio in the above mentioned studies.

In addition, it is widely accepted that there is a correlation between sperm quality and infertility and our results are in accordance with the previous studies (López et al., 2013). In this way, the embryos resulting from morphologically abnormal sperm cells lead to significantly lower pregnancy rates (de Vos et al., 2003). The correlation between spermatozoa with large nuclear vacuoles and ICSI outcomes has been reported (López et al., 2013). While the origin and consequences of vacuoles of sperm head are also a problem of controversy. Therefore, the association among different sizes, locations, and frequencies of vacuole with chromatin status, IVF/ICSI outcomes, and weight of each feature (size, location, and frequency of vacuoles) on pregnancy rate are essential that this study considered them.

Kacem et al. (2010) showed that a large sperm head vacuole could originate from spermatogenesis damaging, abnormal maturation or modifications during the acrosome reaction. Our results are consistent with the results of this study. So that, the immature acrosome reaction was greater in spermatozoa with grade III and IV, therefore, the fertilization rate was decreased in these groups. Of note, to the best of our knowledge, this is the first study in which high magnification with SEM images and deep learning algorithm (Javadi and Mirroshandel, 2019) were used to assess sperm morphology. In addition, sperm parameters, including chromatin structure, chromatin condensation, protamination 
status, acrosome reaction with gene expression involved in chromatin protamination were studied to trace IVF and ICSI outcomes.

\section{Conclusions}

The results of this study indicate that the semen samples from subfertile men are characterized by a higher ratio of vacuolization grades, although there are also in normozoospermia samples. This frequency of vacuolization correlated to abnormal chromatin condensation, greater sperm deprotamination, declined prm1 and prm 2 gene expression, and a high protamine mRNA ratio. Also tracing the IVF/ICSI outcomes shows that the poor fertilization rate during (IVF cycles), embryo quality, and declined clinical pregnancy rate may have related to the abnormal maturation and sperm head vacuoles. Therefore, the evaluation of the vacuole status of semen samples as a definite parameter before starting treatment cycles appears to be a useful technique to introduce the best treatment cycle (IVF or ICSI) in couples undergoing ART.

\section{Abbreviations}

IVF: In-vitro fertilization; ICSI: Intracytoplasmic sperm injection; ART: Assisted reproductive technique; CMA3: Chromomycin A3; q real-time PCR: Real-time quantitative reverse transcription polymerase chain reaction; MSOME: Motile sperm organelle morphology examination; WHO: World Health Organization; SEM: Scanning electron microscopy; TB: Toluidine blue; AB: Aniline blue; PVP: Poly-vinyl pyrrolidone; LNV: Large nuclear vacuole.

\section{Declarations}

\section{Ethics approval and consent to participate}

This study was approved by the Guilan University of Medical Sciences committee. In addition, the informed consent was obtained from all the selected participants in the present study (IR.GUMS.REC.1397.154).

\section{Consent for publication}

Not applicable

\section{Availability of data and materials}

The datasets generated and/or analysed during the current study are not publicly available due [REASON WHY DATA ARE NOT PUBLIC] but are available from the corresponding author on reasonable request.

\section{Competing interests}

The authors declare that they have no competing interests. 


\section{Funding}

This research did not receive any specific grant from funding agencies in the public, commercial, or notfor-profit sectors.

\section{Authors' Contribution}

All authors participated in the design of the study, experiment performance, interpretation, statistical analysis, and reviewing of the manuscript; F.G. and M.H.B. conducted the experiments and manuscript drafting and S.Z.H. and M.E. supplied critical reagents and contribution of patient material/data.

\section{Acknowledgements}

The authors of this study thank for skillful technical assistance (Genetic laboratory, University of Guilan, Rasht, Iran).

\section{References}

Antinori M, Licata E, Dani G, Cerusico F, Versaci C, d'Angelo D, et al. Intracytoplasmic morphologically selected sperm injection: a prospective randomized trial. Reprod Biomed online. 2008; 16: 835-841.

Aoki VW, Liu L, Carrell DT. Identification and evaluation of a novel protamine abnormality in a population of infertile males. Hum Reprod. 2005; 20: 1298-1306.

Aoki VW, Liu L, Jones KP, Hatasaka HH, Gibson M, Peterson CM, et al. Sperm protamine 1/protamine 2 ratios are related to in vitro fertilization pregnancy rates and predictive of fertilization ability. Fertil Steril. 2006; 86: 1408-1415.

Balhorn R, Reed S, Tanphaichitr N. Aberrant protamine 1/protamine 2 ratios in sperm of infertile human males. Experientia. 1988; 44: 52-55.

Bartoov B, Berkovitz A, Eltes F, Kogosowski A, Menezo Y, Barak Y. Real-time fine morphology of motile human sperm cells is associated with IVF-ICSI outcome. J Androl. 2002; 23: 1-8.

Berkovitz A, Eltes F, Ellenbogen A, Peer S, Feldberg D, Bartoov B. Does the presence of nuclear vacuoles in human sperm selected for ICSI affect pregnancy outcome? Hum Reprod. 2006; 21:1787-1790

Berkovitz A, Eltes F, Yaari S, Katz N, Barr I, Fishman A, et al. The morphological normalcy of the sperm nucleus and pregnancy rate of intracytoplasmic injection with morphologically selected sperm. Hum Reprod. 2005; 20:185-190.

Boitrelle F, Ferfouri F, Petit JM, Segretain D, Tourain C, Bergere M, et al. Large human sperm vacuoles observed in motile spermatozoa under high magnification: nuclear thumbprints linked to failure of chromatin condensation. Hum Reprod. 2011; 26: 1650-1658. 
Carrell DT, Liu L. Altered protamine 2 expression is uncommon in donors of known fertility, but common among men with poor fertilizing capacity, and may reflect other abnormalities of spermiogenesis. $J$ Androl. 2001; 4: 604-610.

Cassuto NG, Bouret D, Plouchart JM, Jellad S, Vanderzwalmen P, Balet R, et al. A new real-time morphology classification for human spermatozoa: a link for fertilization and improved embryo quality. Fertil Steril. 2009; 92: 1616-1625.

Cassuto NG, Hazout A, Hammoud I, Balet R, Bouret D, Barak Y, et al. Correlation between DNA defect and sperm-head morphology. Reprod Biomed Online. 2012; 24: 211-218.

de Mateo S, Gazquez C, Guimera M, Balasch J, Meistrich ML, Ballesca JL, et al. Protamine 2 precursors (Pre-P2), protamine 1 to protamine 2 ratio (P1/P2), and assisted reproduction outcome. Fertil Steril. 2009; 91: 715-722.

de Vos A, Van de Velde H, Joris H, Verheyen G, Devroey P, Van Steirteghem A. Influence of individual sperm morphology on fertilization, embryo morphology, and pregnancy outcome of intracytoplasmic sperm injection. Fertil Steril. 2003; 79: 42-48.

Depa-Martynow M, Kempisty B, Jagodzinski PP, Pawelczyk L, Jedrzejcak P. Impact of protamine transcripts and their proteins on the quality and fertilization ability of sperm and the development of preimplantation embryos. Reprod Biol. 2012; 12: 57-72.

Esteves SC, Agarwal A. Novel concepts in male infertility. Int Braz J Urol. 2009; 37: 5-15.

Hammoud S, Liu L, Carrell DT. Protamine ratio and the level of histone retention in sperm selected from a density gradient preparation. Andrologia. 2009; 41: 88-94.

Hazout A, Dumont-Hassan M, Junca AM, Cohen Bacrie P, Tesarik J. High-magnification ICSI overcomes paternal effect resistant to conventional ICSI. Reprod Biomed Online. 2006; 12:19-25.

Javadi S, Mirroshandel SA. A novel deep learning method for automatic assessment of human sperm images. Comput Biol Med. 2019; 109: 182-194.

Kacem O, Sifer C, Barraud-Lange V, Ducot B, de Ziegler D, Poirot C, et al. Sperm nuclear vacuoles, as assessed by motile sperm organellar morphological examination, are mostly of acrosomal origin. Reprod Biomed Online. 2010; 20: 132-137.

Knez K, Tomazevic T, Vrtacnik-Bokal E, Virant-Klun I. Developmental dynamics of IMSI-derived embryos: a time-lapse prospective study. Reprod Biomed Online. 2013; 27(2):161-171.

Knez K, Zorn B, Tomazevic T, Vrtacnik-Bokal E, Virant-Klun I. The IMSI procedure improves poor embryo development in the same infertile couples with poor semen quality: a comparative prospective randomized study. Reprod Biol Endocrinol. 2011; 9:123. 
Larson-Cook KL, Brannian JD, Hansen KA, Kasperson KM, Aamold ET, Evenson DP. Relationship between the outcomes of assisted reproductive techniques and sperm DNA fragmentation as measured by the sperm chromatin structure assay. Fertil Steril. 2003; 80: 895-902.

Lewis SE. Is sperm evaluation useful in predicting human fertility? Reproduction. $2007 ; 134$ : 31-40.

López G, Lafuente R, Checa MA, Carreras R, Brassesco M. Diagnostic value of sperm DNA fragmentation and sperm high-magnification for predicting outcome of assisted reproduction treatment. Asian $\mathrm{J}$ Androl. 2013; 15: 790-794.

Mengual L, Ballesca' JL, Ascaso C, Oliva R. Marked differences in protamine content and P1/P2 ratios in sperm cells from percoll fractions between patients and controls. J Androl. 2003; 3: 438-447.

Nanassy L, Liu L, Griffin J, Carrell DT. The clinical utility of the protamine 1 / protamine 2 ratio in sperm. Protein Pept Lett. 2011; 18; 772-777.

Nasr-Esfahani MH, Salehi M, Razavi S, Mardani M, Bahramian H, Steger K, et al. Effect of protamine-2 deficiency on ICSI outcome. Reprod Biomed Online. 2004; 9; 652-658.

Perdrix A, Travers A, Chelli MH, Escalier D, Do Rego JL, Milazzo JP, et al. Assessment of acrosome and nuclear abnormalities in human spermatozoa with large vacuoles. Hum Reprod. 2011; 26; 47-58.

Rogenhofer N, Dansranjavin T, Schorsch M, Spiess A, Wang H, von Schönfeldt V, et al. The sperm protamine mRNA ratio as a clinical parameter to estimate the fertilizing potential of men taking part in an ART program. Hum Reprod. 2013; 28: 969-978.

Seli E, Gardner DK, Schoolcraft WB, Moffatt O, Sakkas D. Extent of nuclear DNA damage in ejaculated spermatozoa impacts on blastocyst development after in vitro fertilization. Fertil Steril. 2004; 82: 378383.

Sergerie M, Laforest G, Bujan L, Bissonnette F, Bleau G. Sperm DNA fragmentation: threshold value in male fertility. Hum Reprod. 2005; 20: 3446-3451.

Steger K, Cavalcanti MCO, Schuppe HC. Prognostic markers for competent human spermatozoa: fertilizing capacity and contribution to the embryo. Int J Androl. 2011; 34: 513-527.

Talbot P, Chacon RS. A triple-stain technique for evaluating normal acrosome reactions of human sperm. J Exp Zool. 1981; 215: 201-208.

Vanderzwalmen P, Bertin G, Lejeune B, Nijs M, Vandamme B, Schoysman R. Two essential steps for a successful intracytoplasmic sperm injection: injection of immobilized spermatozoa after rupture of the oolemma. Hum Reprod. 1996; 11: 540-547. 
Vanderzwalmen P, Hiemer A, Rubner P, Bach M, Neyer A, Stecher A, et al. Blastocyst development after sperm selection at high magnification is associated with size and number of nuclear vacuoles. Reprod Biomed Online. 2008; 17: 617-627.

World Health Organization. (2010). WHO Laboratory Manual for the Examination and Processing of Human Semen, 5th edn Geneva: WHO Press. 2010.

\section{Figures}

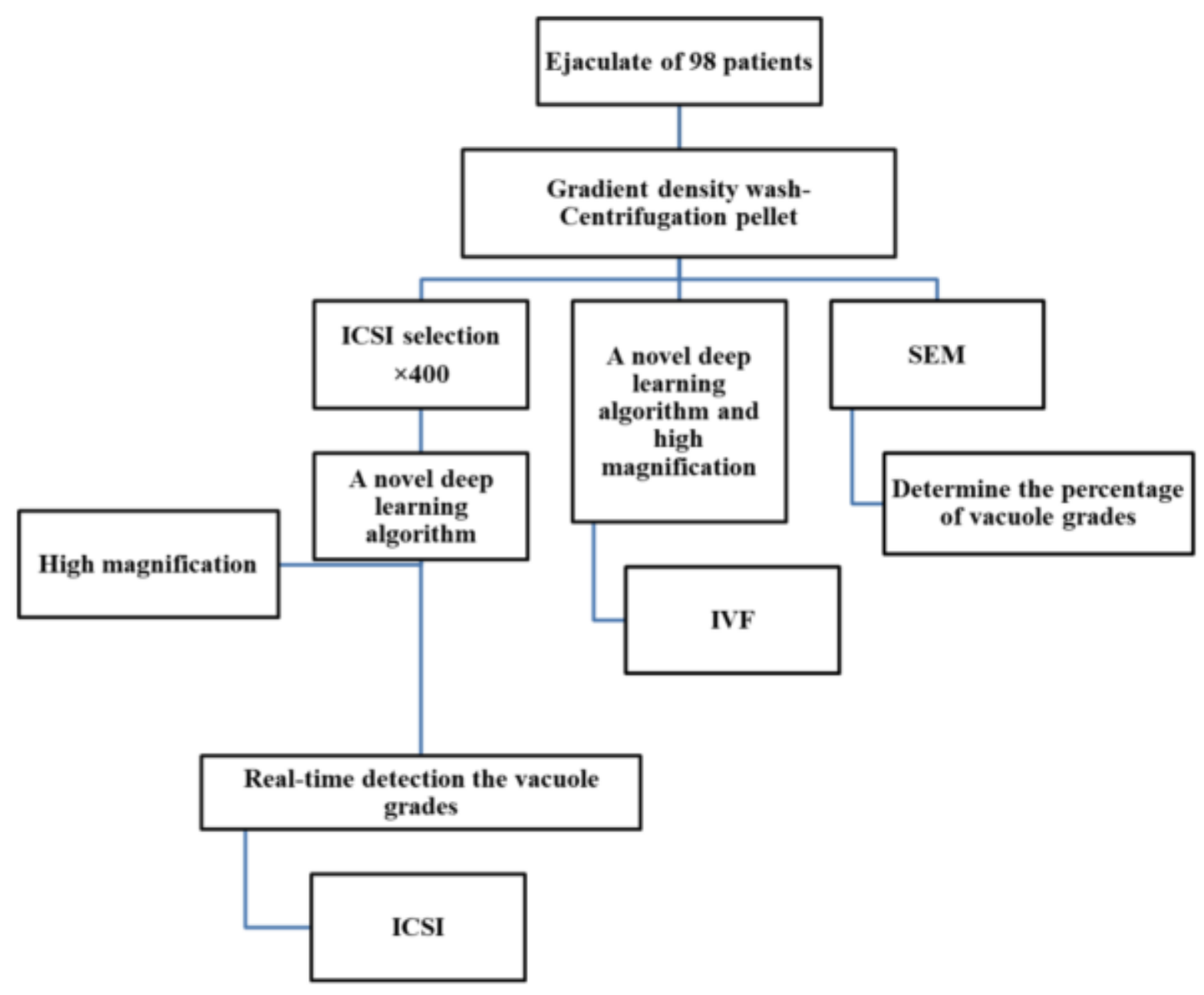

Figure 2

Experimental design. The selection of spermatozoa with different grades (I, II, III, and IV) at $\times 400$ magnification using a novel deep learning algorithm in relation to the the percentage of normal/abnormal spermatozoa assessed during ICSI and IVF as real-time. ICSI: Intracytoplasmic sperm injection; IVF: invitro fertilization; SEM: Scanning electron microscope. 

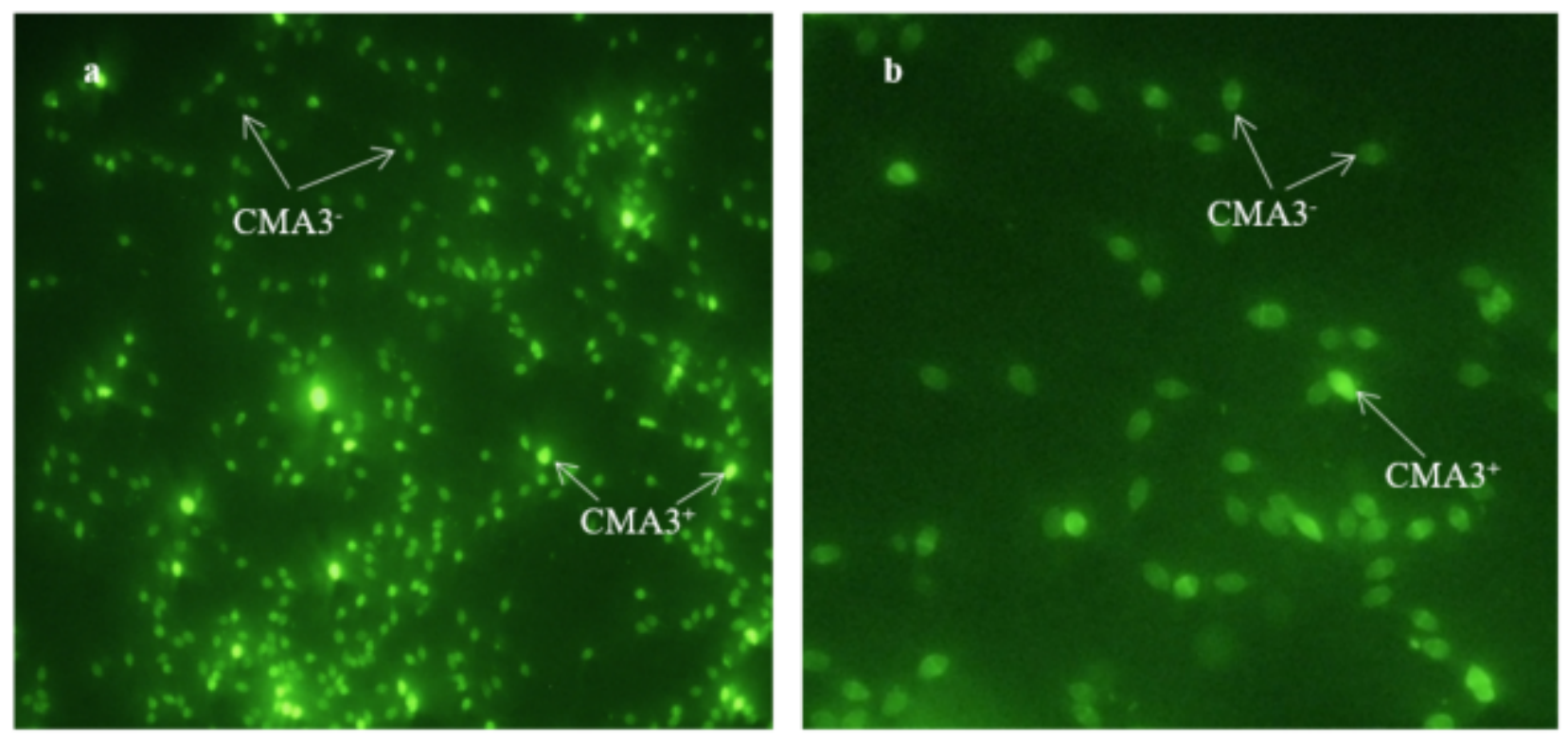

\section{Figure 4}

Sperm chromatin deprotamination. Spermatozoa with dull yellow/normal chromatin (CMA3-) and bright yellow/abnormal chromatin (CMA3+) $(a: \times 10$ and $b: \times 40)$.
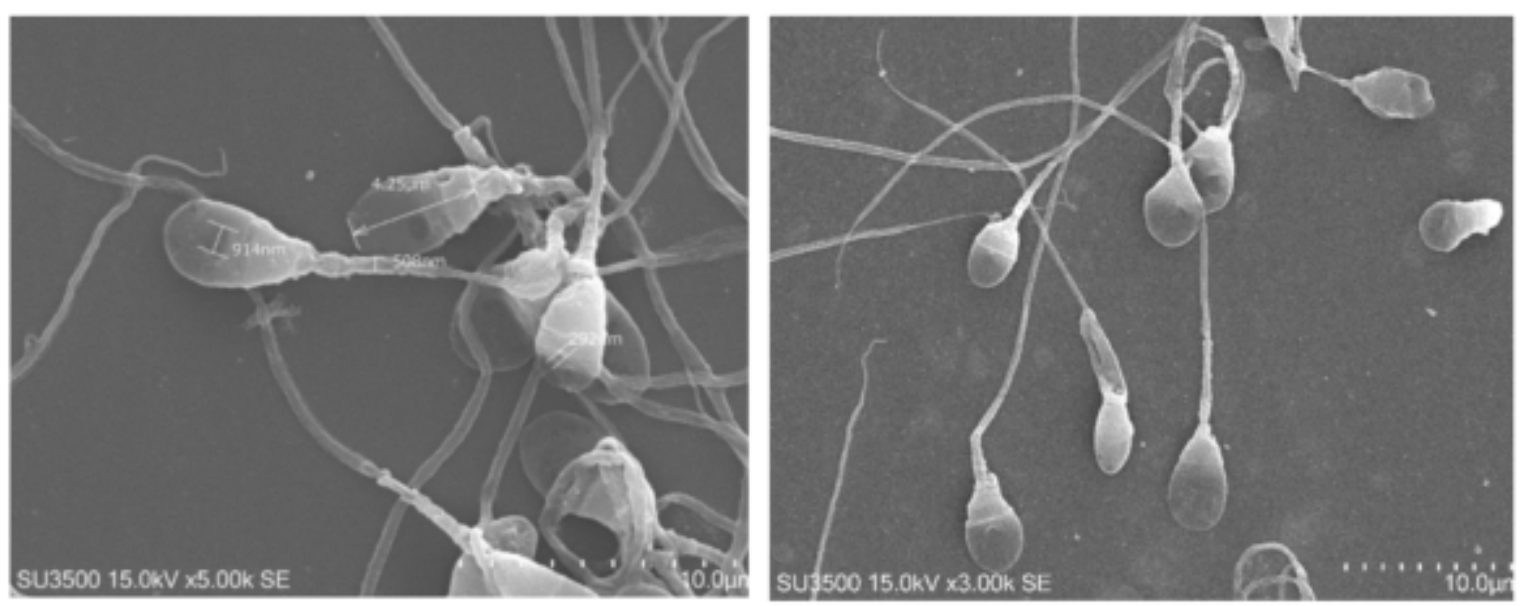

\section{Figure 5}

Scanning electron microscope images of vacuolated spermatozoa. The presence of small and large vacuoles, its frequency and located in the nuclear or non-nuclear position is clear. 

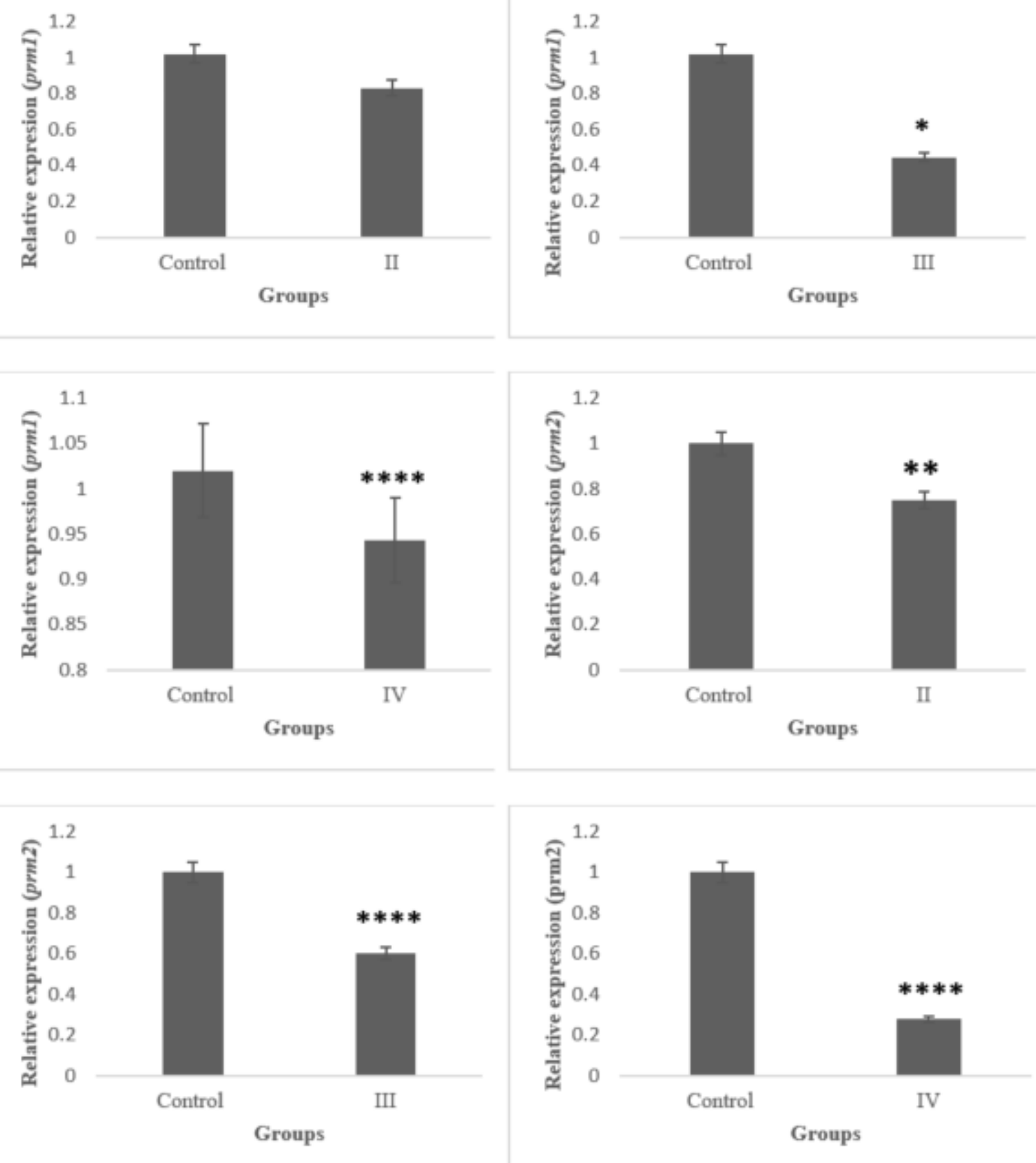

\section{Figure 7}

The protamine 1 and protamine 2 gene expression of vacuolated spermatozoa from subfertile men. A significant difference can be seen in the prm1 gene expression (underexpression) of spermatozoa with grades of III ( $p<0.05)$ and IV ( $<<0.0001)$ compared to control group. There is significant difference in the prm2 gene expression (over-expression) from spermatozoa with grades of II $(p<0.01)$, III $(p<0.0001)$, and IV $(p<0.0001)$ in comparison to control group. $*<0.05, * *<0.01, * \star *<0.001$, and $* * \star *<0.001$. 